\title{
Schwerpunkt: Auswirkungen frühpädagogischer Institutionen auf die kindliche Entwicklung
}

\author{
Hans-Günther Roßbach · Jürgen Baumert
}

Schon seit einiger Zeit ist in Deutschland ein gestiegenes öffentliches und politisches Interesse an frühkindlicher Bildung zu verzeichnen. Die Bemühungen um eine verbesserte Vereinbarkeit von Familie und Beruf, der ab dem 1. August 2013 bestehende Rechtsanspruch auf einen Betreuungsplatz in Kindertageseinrichtungen oder Kindertagespflege für Kinder ab dem vollendeten ersten Lebensjahr, frühe Defizite in der Beherrschung der Verkehrssprache Deutsch, vor allem bei Kindern aus Familien mit einer nicht-deutschen Herkunftssprache, der offensichtlich nur eingeschränkte Erfolg von Sprachförderprogrammen für diese Adressatengruppe, die Notwendigkeit der Kontrolle und Verbesserung der pädagogischen Qualität in den Einrichtungen und vor allem auch die Erwartungen an die förderlichen Auswirkungen des Besuchs einer frühpädagogischen Einrichtung für alle Kindern und in besonderem Maße für Kinder aus benachteiligten Familien stellen große Herausforderungen an das gesamte System der Bildung, Erziehung und Betreuung von Kindern vor Schulbeginn. Fragen der frühpädagogischen Förderung in Institutionen hat sich die ZfE bereits mit ihrem Sonderheft 11 im Jahr 2008 zugewandt. Während dort eine breite Perspektive eröffnet wurde, unter der neben Auswirkungen des Besuchs frühpädagogischer Einrichtungen vor allem soziale Disparitäten bei der Nutzung der Einrichtungen, Fragen der Steuerung des frühpädagogischen Systems und die empirische Überprüfung von pädagogischen Programmen in den Blick genommen wurden, berichtet der vorliegende Schwerpunkt speziell über den Stand der Forschung zu den Auswirkungen des Besuchs frühpädagogischer Einrichtungen.

Der erste Beitrag dieses Schwerpunktes von Yvonne Anders gibt einen systematischen Überblick über den internationalen Forschungsstand. Die Autorin arbeitet heraus, dass es nicht die Auswirkungen gibt, sondern dass sorgfältig zwischen den in den Studien unter-

Online publiziert: 29.06 .2013

(C) Springer Fachmedien Wiesbaden 2013

Prof. Dr. H.-G. Roßbach $(\bowtie)$

Otto-Friedrich-Universität Bamberg, 96045 Bamberg, Deutschland

E-Mail: hans-guenther.rossbach@uni-bamberg.de

Prof. Dr. J. Baumert

Max-Planck-Institut für Bildungsforschung,

Lentzeallee 94, 14195 Berlin, Deutschland

E-Mail: sekbaumert@mpib-berlin.mpg.de 
suchten Aspekten differenziert werden muss. Eine erste Unterscheidung betrifft das Alter der Kinder. Hier zeigen sich durchaus unterschiedliche Auswirkungen je nachdem, ob jüngere Kinder (etwa unter drei Jahren) oder ältere Kinder (ab etwa drei Jahren) untersucht werden. Unterschiedliche Ergebnisse finden sich auch in Abhängigkeit davon, ob die „Dosis“ des Besuchs einer frühpädagogischen Einrichtung (z. B. Dauer in Monaten, Stunden pro Woche, erstmaliger Beginn des Besuch einer Einrichtung) oder die „Qualität"“ der in den Einrichtungen erfahrenen Anregung und Unterstützung betrachtet werden. Ferner ist zu berücksichtigen, ob es sich um Erfahrungen in Regeleinrichtungen oder um spezifische Modellprogramme (meistens für Kinder aus benachteiligten Familien) handelt. Weiterhin muss differenziert werden, in welchen Dimensionen Auswirkungen erwartet werden. Die Autorin unterscheidet hier Auswirkungen auf die kognitiv-leistungsbezogene und auf die sozio-emotionale Entwicklung sowie - was seltener berücksichtigt wird - Auswirkungen auf allgemeine Indikatoren der Lebensbewältigung wie z.B. die Schulkarriere. Letzteres ist auch deshalb bedeutsam, weil gerade in deutschen Einrichtungen ein wichtiges Ziel der Frühpädagogik die Bewältigung der aktuellen und zukünftigen Lebenssituationen der Kinder ist. Systematisch wird auch auf die Frage eingegangen, ob Kinder aus bildungsbenachteiligten Familien in einem besonderen Maße von der Quantität und Qualität der Erfahrungen in Kindertageseinrichtungen profitieren (kompensatorische Effekte). Etwas vereinfacht kann man festhalten, dass sich für alle Kinder positive Effekte des Besuchs einer frühpädagogischen Einrichtung zeigen. Für Kinder aus bildungsbenachteiligten Familien scheint vor allem ein früher Eintritt in eine Einrichtung förderlich zu sein, während nicht gesichert ist, ob sie auch von eine hohen Qualität in besonderer Weise profitieren.

Der zweite Beitrag von Kathy Sylva, Pam Sammons, Lydia L. S. Chan, Edward Melhuish, Iram Siraj-Blatchford und Brenda Taggart wendet sich einer international sehr beachteten Längsschnittstudie in England zu, dem Effective Pre-School, Primary and Secondary Education Research Project (EPPSE). Diese Längsschnittstudie begann 1997 mit über 3.000 dreijährigen Kindern aus 141 frühpädagogischen Einrichtungen (PreSchools) unterschiedlichen Typs. Zu Beginn waren die Kinder etwa drei Jahre alt und in der Regel im ersten Jahr ihres Besuchs einer Pre-School. Die Kinder wurden während ihrer gesamten Pflichtschulzeit und darüber hinaus (also über das 16. Lebensjahr hinaus) weiterverfolgt. Die Studie hatte auch einen erheblichen Einfluss auf politische Maßnahmen zur Ausgestaltung der Frühförderung in England. Der vorliegende Beitrag der Autoren berichtet über die Auswirkungen der Anregungsqualität in den Familien (Home Learning Environment - HLE) und Pre-Schools auf die spätere Entwicklung von akademischen Fähigkeiten (Englisch und Mathematik) im Alter von sieben bis elf Jahren. Unter Kontrolle anderer Einflussfaktoren finden sich positive Beziehungen zwischen der vorschulischen Anregungen in Familie und Pre-School einerseits und sowohl dem Leistungsstand in Englisch und Mathematik im Alter von elf Jahren als auch andererseits der Leistungsentwicklung vom siebten bis zum elften Lebensjahr (Jahrgangsstufe 2 bis 6). Die Autoren schließen, dass die „Benefits“ der vorschulischen Erfahrungen in Familie und Einrichtung langandauernd und kumulativ sind. Als praktische Konsequenz schlagen sie vor: ein universelles Angebot in frühpädagogischen Einrichtungen für alle Kinder im Alter von drei und vier Jahren, das eine hohe Förderqualität sicherstellt, sowie eine Unterstützung der Familien durch Elternbildung und familiale Förderprogramme. 
Der dritte Beitrag von Sabine Weinert und Susanne Ebert berichtet aus der interdisziplinären Bamberger DFG-Forschergruppe „BiKS - Bildungsprozesse, Kompetenzentwicklung und Formation von Selektionsentscheidungen im Vorschul- und Grundschulalter". In dem Längsschnitt BiKS-3-10 wurden von 2005 an 547 Kinder aus 97 Kindertageseinrichtungen in Bayern und Hessen längsschnittlich bis (gegenwärtig) zum zehnten Lebensjahr verfolgt. Die Autorinnen beschränken sich in zwei Studien auf den Spracherwerb während der Kindergartenzeit von - in der Regel - dem ersten bis zum letzten Kindergartenjahr. Zentrale Befunde der ersten Studie sind, dass sich bereits im Alter von drei Jahren soziale Disparitäten im Wortschatz und in grammatischen Kompetenzen in Abhängigkeit vom sozialen Hintergrund der Familie zeigen und dass keine substanziellen Veränderungen dieser Disparitäten über die Kindergartenzeit feststellbar sind. Die zweite Studie betrachtet die Entwicklung grammatikalischer Kompetenzen während der Kindergartenzeit. Während familiale Merkmale mit den frühen grammatikalischen Kompetenzen zusammenhängen, ist dies für Qualitätsmerkmale der besuchten Kindergärten nicht der Fall. Als mögliche Erklärungen nennen die Autorinnen u. a. die insgesamt niedrige bereichsspezifische Förderqualität in den untersuchten Kindergärten sowie die eher geringen Qualitätsunterschiede zwischen den Einrichtungen.

C. Katharina Spieß betrachtet im vierten Beitrag die Auswirkungen frühkindlicher Bildungs- und Betreuungsprogramme aus einer bildungsökonomischen Perspektive. Nach einer Beschreibung von drei grundsätzlichen Ansätzen für Effizienzanalysen und einer Darstellung der möglicherweise zwischen Analysen variierenden Parameter von Kosten-Nutzen-Analysen stellt sie drei Beispiel solcher Analysen aus den USA vor (die auch schon in dem Beitrag von Yvonne Anders berücksichtigt wurden, allerdings nicht unter bildungsökonomischer Perspektive). Alle drei Kosten-Nutzen-Analysen, bei denen der Nutzen die Kosten zum Teil sehr deutlich übersteigt, haben in der Diskussion um die Implementation spezieller Förderprogramme für Kinder aus sozial benachteiligten Familien und ihre finanzielle Förderung mit öffentlichen Mitteln eine bedeutsame und unterstützende Rolle in den USA gespielt. Vor diesem Hintergrund diskutiert die Autorin Möglichkeiten für Kosten-Nutzen-Analysen in Deutschland, hält aber auch fest, dass solche Analysen andere pädagogisch-psychologisch orientierte Analysen der Auswirkungen des Besuchs frühpädagogischer Einrichtungen nicht ersetzen, sondern ergänzen.

Über geschichtliche Aspekte der Früherziehung berichtet auch Frithjof Grell in seiner Sammelrezension „Historische Frühpädagogik“. 